\title{
ESTIMAÇÃO DE FREQUÊNCIA USANDO SENSORES DE ERRO COM ATRASOS ADAPTATIVOS
}

\author{
Diego Paolo Ferruzzo Correa* \\ diegopaolo@lac.usp.br
}

Átila Madureira Bueno ${ }^{\dagger}$

atilaelac.usp.br

\author{
José Roberto Castilho Piqueira* \\ piqueira@lac.usp.br \\ *Escola Politécnica da Universidade de São Paulo \\ Avenida Prof. Luciano Gualberto, travessa 3, n. 158, 05508-900, São Paulo, SP, Brasil. \\ Phone: (55)(11) 30915464 - 30915647 \\ ${ }^{\dagger}$ Universidade Estadual Paulista - UNESP \\ Departamento de Estatística, Matemática Aplicada e Computação. \\ Rio Claro - SP
}

\section{ABSTRACT}

Frequency estimation by using error sensors with adaptive delays

This paper proposes a solution to improve the performance of the first order Early Error Sensing (EES) Adaptive Time Delay Tanlock Loops (ATDTL) presented in (Al-Zaabi, AlQutayri e Al-Araji, 2005), regarding to frequency estimation and tracking time. The EES-ATDTL are phaselocked-loops (PLL) used to hardware implementations, due to their simple structure. Fixed-points theorems are used to determine conditions for rapid convergence of the estimation process and a estimative of the frecuency input is obtained with a Gaussian filter that improves the gain adaptation. The mathematical models are the presented by (Al-Araji, Al-Qutayri e Al-Zaabi, 2006). Simulations have been performed to evaluate the theoretical results.

KEYWORDS: Early Error Sensing Time Delay Tan-lock Loop, Gaussian filter, adaptive gain, digital PLL, synchronism, fixed-point theorem.

Artigo submetido em 06/12/2010 (Id.: 01229)

Revisado em 15/03/2011, 22/03/2011, 25/03/2011

Aceito sob recomendação do Editor Associado Prof. Daniel Coutinho

\section{RESUMO}

Neste trabalho é proposta uma solução para a melhoria no desempenho dos Early Error Sensing (EES) Adaptive Time Delay Tanlock Loops (ATDTL) de $1^{\text {a }}$ ordem apresentados em (Al-Zaabi et al., 2005), quanto a estimação de frequência e tempo de captura. Os EES-ATDTL são Phase-Locked Loops (PLL) digitais utilizados para implementações em hardware, devido à simplicidade da sua estrutura. Teoremas de ponto fixo são usados para se determinar condições sob as quais é possível obter uma convergência rápida no processo de captura e é obtida uma estimativa da frequência de entrada, utilizando um filtro de tipo Gaussiano para melhorar a adaptação do ganho. Os modelos matemáticos usados são os apresentados em (Al-Araji et al., 2006). São feitas simulações para avaliar os resultados teóricos.

PALAVRAS-CHAVE: Early Error Sensing Time Delay Tanlock Loop, filtro Gaussiano, ganho adaptativo, PLL digital, sincronismo, teorema do ponto fixo.

\section{INTRODUÇÃO}

O estudo dos PLL analógicos vem sendo desenvolvido com diferentes perspectivas nos anos recentes, desde os trabalhos de Piqueira em (Piqueira, 1997), com enfoque nas 
respostas das diferentes arquiteturas para entradas de processo de fase diversos, e os métodos para o cálculo das taxas de falha de sincronização de Popken em (Popken, Kriedte, Bender e Luise, 1993), passando pela estimação do número crítico de nós escravos em uma rede de via única proposta por Monteiro em (Monteiro, Dos Santos e Piqueira, 2003), e pela determinação do sincronismo em redes mestre-escravo e mutuamente conectadas com e sem atraso em (Monteiro, Garcia e Piqueira, 2003), (Bueno, Rigon e Ferreira, 2010), (Bueno, Ferreira e Piqueira, 2010), (Bueno, Ferreira e Piqueira, 2009).

No caso dos PLL digitais, têm se estudado métodos de otimização, considerando redes de PLLs e a sua interconexão (Orsatti, Carareto e Piqueira, 2008), (Carareto, Orsatti e Piqueira, 2009). Também se têm analisado o ruído e a sincronização de redes de PLLs totalmente digitais (Vamvakos, Staszewski, Sheba e Waheed, 2006), (Akre and, Juillard, Galayko e Colinet, 2010).

Nas últimas três décadas, os PLL totalmente digitais têm recebido muita atenção devido, principalmente, às vantagens que eles oferecem em comparação aos PLL analógicos. Essas vantagens incluem melhor desempenho, maior velocidade e confiabilidade, tamanho reduzido e menor custo (Saleh R. Al-Araji e Al-Qutayri, 2006). Eles também são amplamente utilizados em sistemas de comunicações e em outras aplicações digitais (Best, 2003).

O presente estudo pretende ser uma contribuição no campo dos PLL digitais, otimizados para a sua implementação em hardware, fazendo uma análise de suas caraterísticas dinâmicas e propondo uma nova estrutura, capaz de melhorar a resposta transitória da malha.

Os Time Delay Tanlock Loop ou TDTL, apresentados em (Hussain, Boashash, Hassan-Ali e Al-Araji, 2001), são um tipo novo de PLL digital, amostrado não uniformemente, que utiliza um atraso constante, ao contrário dos Digital Tanlock Loop ou DTL apresentados em (Lindsey e Chie, 1981), que utilizam uma transformação de Hilbert (Lee e Un, 1982). Os TDTL apresentam uma melhora da faixa de captura e simplicidade para a sua implementação, quando comparados com os DTL, considerando a dificuldade para sintetizar em hardware o bloco de Hilbert.

No entanto, os TDTL não conservam a linearidade na saída do detector de fase, não sendo possível utilizar a teoria clássica dos sistemas lineares discretos na sua análise. Ao invés disso, utiliza-se a análise de mapas contrativos e pontos fixos, cujos teoremas, em versão resumida, encontram-se em (Osborne, 1980).

Na figura 1 é apresentado um TDTL com deteção rápida de frequência e ganho adaptativo, nomeado de EES-

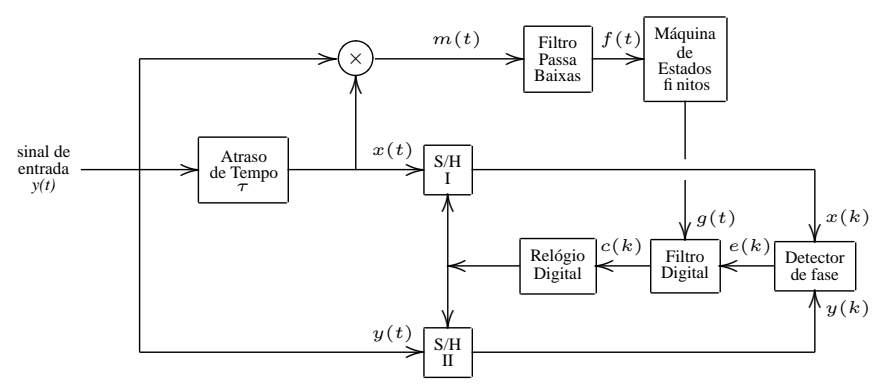

Figura 1: Diagrama de blocos do EES-ATDTL.

ATDTL (Al-Zaabi et al., 2005), com a idéia principal de se ajustar o ganho proporcional do filtro digital a um valor que garanta a sincronização caso a frequência de entrada, quando comparada com a frequência do relógio digital, esteja fora da faixa de captura.

A arquitetura proposta melhora o desempenho o EESATDTL em dois aspectos: o primeiro é no sentido de obter uma aproximação da frequência do sinal de entrada, e o segundo é de adaptar o ganho do filtro digital, não apenas para garantir sincronização, mas, também, para que essa seja mais rápida.

\section{MODELO MATEMÁTICO}

Para desenvolver o modelo matemático do EES-ATDTL mostrado na figura 1, a linha proposta em (Hussain et al., 2001) é utilizada.

O TDTL recebe em sua entrada um sinal senoidal $y(t)$, contínuo no tempo:

$$
\begin{gathered}
y(t)=A \sin \left[\omega_{0} t+\theta(t)\right] \\
\theta(t)=\Delta \omega t+\theta_{0},
\end{gathered}
$$

sendo $A$ a amplitude do sinal, $\omega_{0}$ a frequência angular de oscilação de livre curso do relógio digital do TDTL e $\theta(t)$ o processo de fase, que depende da diferença de frequências entre o sinal de entrada e o sinal gerado pelo relógio $(\Delta \omega=$ $\left.\omega-\omega_{0}\right)$ e de $\theta_{0}$, que é a fase inicial.

O atraso $\tau$ produz uma desfasagem $\psi$ do sinal $x(t)$ em relação ao sinal $y(t)$, igual a $\omega \tau$, tal que:

$$
x(t)=A \sin \left[\omega_{0} t+\theta(t)-\psi\right] .
$$

No $k$-ésimo instante de amostragem, os valores $y(k)$ e $x(k)$ são:

$$
\begin{gathered}
y(k)=A \sin \left[\omega_{0} t(k)+\theta(k)\right], \\
x(k)=A \sin \left[\omega_{0} t(k)+\theta(k)-\psi\right],
\end{gathered}
$$


sendo $\theta(k)$ igual a $\theta[t(k)]$.

O intervalo de tempo entre os instantes de amostragem $t(k)$ e $t(k-1)$ é uma função da saída do filtro digital dada por:

$$
T(k)=T_{0}-c(k-1),
$$

com $T_{0}$ sendo o período da oscilação de livre curso do oscilador local $\left(T_{0}=\frac{2 \pi}{\omega_{0}}\right)$, e $c(i)$, a saída do filtro digital no $i$-ésimo instante de amostragem. Assumindo que $t(0)=0$, o tempo total $t(k)$ até o $k$-ésimo instante de amostragem é:

$$
t(k)=\sum_{i=0}^{k} T(i)=k T_{0}-\sum_{i=0}^{k-1} c(i) .
$$

Substituindo a equação 7 nas equações 4 e 5, têm-se:

$$
y(k)=A \sin \left[\theta(k)-\omega_{0} \sum_{i=0}^{k-1} c(i)\right]
$$

$\mathrm{e}$

$$
x(k)=A \sin \left[\theta(k)-\omega_{0} \sum_{i=0}^{k-1} c(i)-\psi\right] .
$$

Definindo o erro de fase como:

$$
\phi(k)=\theta(k)-\omega_{0} \sum_{i=0}^{k-1} c(i)-\psi,
$$

pode-se expressar $y(k)$ e $x(k)$ como:

$$
y(k)=A \sin [\phi(k)+\psi]
$$

$\mathrm{e}$

$$
x(k)=A \sin [\phi(k)] .
$$

A partir das equações 2,7 e 10, escreve-se:

$$
\phi(k+1)=\phi(k)-\omega c(k)+\Lambda_{0},
$$

$\operatorname{com} \Lambda_{0}$ igual a $2 \pi \frac{\omega-\omega_{0}}{\omega_{0}}$.

A equação 13 é o modelo matemático do TDTL.

A saída do detector de fase $e(k)$ é obtida a partir do quociente dos sinais $x(k)$ e $y(k)$, usando a função ${ }^{1} \operatorname{Tan}^{-1}\left(\frac{x(k)}{y(k)}\right)$ :

$$
e(k)=f\left[\operatorname{Tan}^{-1}\left(\frac{\sin \{\phi(k)\}}{\sin \{\phi(k)+\psi\}}\right)\right],
$$

sendo $f[\alpha]=-\pi+\{(\alpha+\pi)$ modulo $(2 \pi)\}$, com $|f[\alpha]|<\pi$.

\footnotetext{
${ }^{1}$ Diferentemente da função clássica $\tan ^{-1}(\cdot)$, que é defi nida apenas no intervalo $\left(-\frac{\pi}{2}, \frac{\pi}{2}\right)$, a função $\operatorname{Tan}^{-1}(\cdot)$ pode ser defi nida em todo o intervalo $(0,2 \pi)$, dado que são conhecidos os sinais de $x(k)$ e $y(k)$, em cada instante de amostragem.
}

A função característica do detector de fase pode ser expressa em termos da desfasagem nominal ${ }^{2}\left(\psi_{0} \triangleq \omega_{0} \tau\right)$ e da frequência normalizada $\left(W \triangleq \frac{\omega_{0}}{\omega}\right)$, assim:

$$
h_{\psi}(\phi)=f\left[\operatorname{Tan}^{-1}\left(\frac{\sin (\phi)}{\sin \left(\phi+\frac{\psi_{0}}{W}\right)}\right)\right] .
$$

\section{O TDTL DE 1a ORDEM}

No presente trabalho, analisa-se o TDTL de primeira ordem com entrada tipo degrau em frequência, logo o filtro digital $D(z)$ é apenas um ganho $G_{1}$ assumido positivo e constante. Então, a partir da equação 13, obtém-se:

$$
\phi(k+1)=\phi(k)-K_{1}^{\prime} h_{\psi}[\phi(k)]+\Lambda_{0} .
$$

Já que $K_{1}^{\prime}$ é igual a $\omega G_{1}$, pode-se definir $K_{1}$ como $\omega_{0} G_{1}$, e com isso $K_{1}^{\prime}$ é, também, $K_{1} / W$.

A equação 16, pode ser expressa como um mapa $\phi \mapsto g(\phi)$, $\phi \in \mathbb{R}$, tal que a função:

$$
g(\phi)=\phi-K_{1}^{\prime} h_{\psi}(\phi)+\Lambda_{0},
$$

apresente um ponto fixo em $\phi_{s s}$, que seja uma solução da equação 17, da seguinte forma (Wiggins, 1990):

$$
g\left(\phi_{s s}\right)=\phi_{s s} .
$$

A sequência $\{\phi(k)\}$ definida na equação 16 convergirá localmente a $\phi_{s s}$ (Osborne, 1980), quando $k \rightarrow \infty$, se:

$$
\left|g^{\prime}\left(\phi_{s s}\right)\right|<1 \text {. }
$$

Sabendo que $h_{\psi}(\phi)$ é contínua e diferenciável no intervalo $(-\pi, \pi)$ (Saleh R. Al-Araji e Al-Qutayri, 2006), pode-se garantir que $g\left(\phi_{s s}\right)$ também é contínua e diferenciável no mesmo intervalo.

Agora, a partir das equações 14 e 17, a saída do detector de fase no estado estacionário $e_{s s}$ é dada por:

$$
e_{s s}=f\left[\operatorname{Tan}^{-1}\left(\frac{\sin \left(\phi_{s s}\right)}{\sin \left(\phi_{s s}+\psi\right)}\right)\right]=\frac{\Lambda_{0}}{K_{1}^{\prime}},
$$

como $|f[\cdot]|<\pi$ :

$$
\left|\frac{\Lambda_{0}}{K_{1}^{\prime}}\right|<\pi
$$

Da equação 20 pode-se obter:

$$
\tan \left(\phi_{s s}\right)=\frac{\sin (\psi) \tan (\eta)}{1-\cos (\psi) \tan (\eta)},
$$

\footnotetext{
${ }^{2} \mathrm{O}$ deslocamento de fase nominal $\psi_{0}$ é o atraso de fase medido em relação ao sinal gerado no relógio digital local, tal que $\psi=\psi_{0} / W$.
} 
com $\eta$ igual a $\Lambda_{0} / K_{1}^{\prime}$.

Definindo:

$$
\beta=\frac{\sin (\psi) \tan (\eta)}{1-\cos (\psi) \tan (\eta)}=\frac{\sin (\psi)}{\cot (\eta)-\cos (\psi)}
$$

e

$$
\alpha=\tan ^{-1}(\beta),
$$

com $\tan ^{-1}(\cdot)$ contida no intervalo $\left(-\frac{\pi}{2}, \frac{\pi}{2}\right)$, tem-se:

$$
\phi_{s s}=\alpha+m \pi, \quad m \in\{-1,0,1\} \text {, }
$$

sendo $m \pi$ o fator de correção devido à $\tan (\cdot)$, tomada na equação 22 .

Das equações 17, 19 e 24, obtém-se:

$$
\left|1-\frac{K_{1}^{\prime} \sin (\psi)}{\sin ^{2}(\alpha)+\sin ^{2}(\alpha+\psi)}\right|<1 .
$$

Das inequações 21 e 26, pode-se estabelecer a faixa de captura para o TDTL de primeira ordem em termos de $K_{1}$ e $W$ :

$$
2|1-W|<K_{1}<2 W \frac{\sin ^{2}(\alpha)+\sin \left(\alpha+\psi_{0} / W\right)}{\sin \left(\psi_{0} / W\right)},
$$

que pode ser resolvida numericamente para diferentes valores de $\psi_{0}$, (Fig. 2).

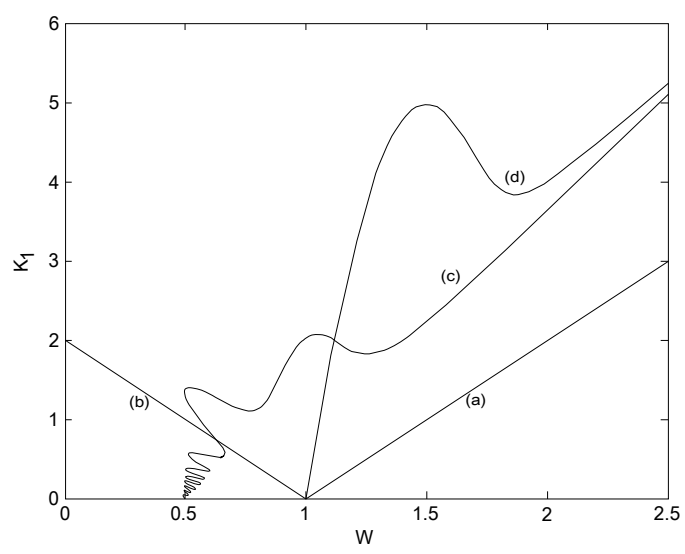

Figura 2: Faixas de capturas para o TDTL de primera ordem para diferentes valores de $\psi_{0}$. A região delimitada pelas curvas (a) e (d) corresponde a $\psi_{0}=\pi$, a região delimitada pelas curvas (a), (b) e (c) corresponde a $\psi_{0}=\pi / 2$.

O valor do erro de estado estacionário para o TDTL de $1^{\mathrm{a}}$ ordem pode ser calculado a partir da equação 20, levando em conta o sinal de $\eta\left(=\Lambda_{0} / K_{1}^{\prime}\right)$. Segundo (Saleh R. Al-Araji e Al-Qutayri, 2006), o dito erro pode ser expresso como:

$$
\phi_{s s}=\left\{\begin{array}{cc}
\alpha & \beta \sin (\eta) \geq 0 \\
f[\alpha+\pi] & \text { caso contrário. }
\end{array}\right.
$$

O funcionamento do EES-ATDTL, tal como proposto em (Al-Zaabi et al., 2005), apresentado na figura 1, pode ser entendido do seguinte modo: na figura 3, suponha que $B$ é o ponto de operação inicial, com $W=1$ e $K_{1}=0$, 5; escolhese o dito valor de $K_{1}$ para se garantir uma boa relação sinalruído segundo (Hussain e Boashash, 2001). Quando o sinal de entrada muda o ponto de operação para $A$ (ou $C$ ), fora da faixa de captura, o TDTL não é capaz de seguir a frequência de entrada, sob tais condições uma máquina de estados finitos muda o ganho do filtro digital, mudando o ponto de operação para $D$ (ou $E$ ), de modo que, com o novo ganho, o TDTL possa atingir o sincronismo.

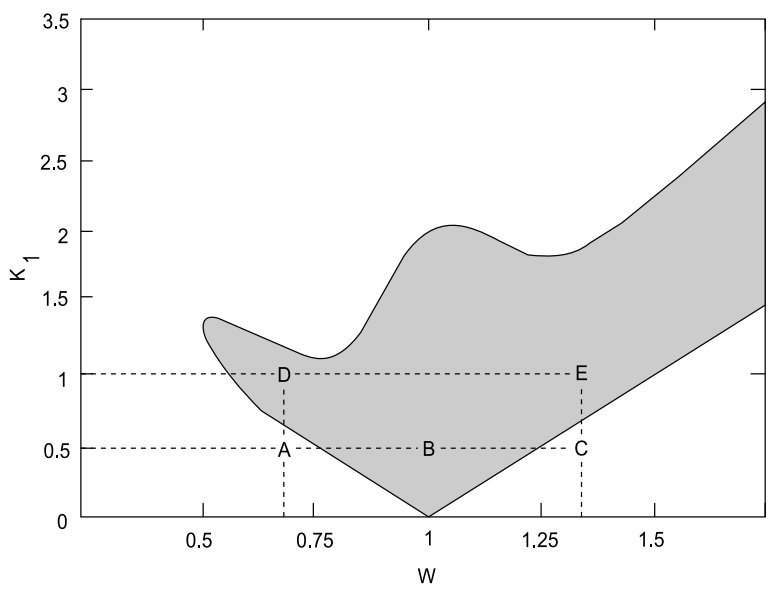

Figura 3: A região sombreada representa a faixa de captura para o TDTL de $1^{\text {a }}$ ordem com $\psi_{0}=\pi / 2$. Os pontos A e C ficam fora da faixa.

\section{ESTIMATIVA DE FREQUÊNCIA}

Para se obter uma estimativa da frequência do sinal de entrada, toma-se como referência o trabalho de Al-araji em (AlZaabi et al., 2005) e (Saleh R. Al-Araji e Al-Qutayri, 2006), baseado no produto $m(t)$ dos sinais $y(t)$ e $x(t)$. Então, das equações 1 e 3 :

$$
\begin{aligned}
m(t) & =x(t) \cdot y(t) \\
& =\left(A^{2} / 2\right)\left[\cos (\psi)-\cos \left(2 \omega_{0} t+2 \theta(t)-\psi\right)\right] .
\end{aligned}
$$

A equação 29 mostra que é possível filtrar o termo de frequência dupla, a fim de se obter uma estimativa da frequência de entrada $\omega$, a partir da obtenção de $\cos (\psi)$, já que $\omega=\psi / \tau$.

Portanto, se escolhe um filtro passa baixas com frequência de corte ajustada para eliminar o termo de frequência dupla do produto $m(t)$, de modo que a sua saída $f(t)$ seja propor- 
cional apenas a $\cos (\psi)$ :

$$
f(t)=\left(A^{2} / 2\right) \cos (\psi) .
$$

Restringe-se os valores de $\psi$ ao intervalo $(0, \pi)$ a fim garantir a invertibilidade da função $\cos (\psi)$, portanto:

$$
0<\psi<\pi
$$

ou

$$
0<\psi_{0} / W<\pi
$$

Das faixas de captura para o TDTL de $1^{\mathrm{a}}$ ordem mostradas nas figuras 2 e 3, pode-se ver que as frequências admissíveis são menores que o dobro da frequência do oscilador local, isto é $W>0,5$. Portanto, a partir da equação 32 se pode estabelecer uma restrição para $\psi_{0}$ :

$$
0<\psi_{0} \leq \pi / 2 .
$$

Se a restrição 33 se cumprir, pode-se calcular a frequência $\bar{\omega}$, como uma aproximação da frequência de entrada $\omega$, usando a equação 30 :

$$
\bar{\omega}=\frac{1}{\tau} \cos ^{-1}\left[\frac{2 f(t)}{A^{2}}\right] .
$$

A estimativa $\bar{\omega}$ está sujeita ao tipo de resposta ao degrau do filtro passa baixas, o qual pode ser escolhido para satisfazer os requisitos de sobressinal e tempo de acomodação desejados. Nota-se a dependência de $\bar{\omega}$ com o quadrado da amplitude $A$ do sinal de entrada.

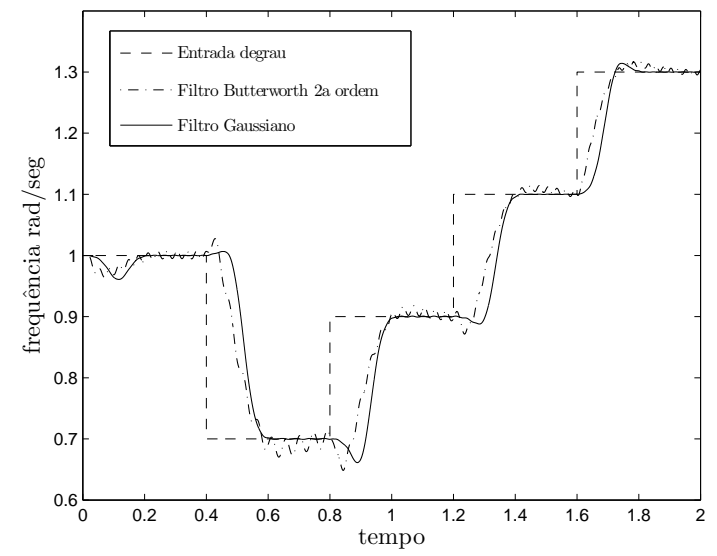

Figura 4: Frequência de entrada ao TDTL e frequências estimadas por meio dos filtros Passa baixas Butterworth de $2^{\mathrm{a}}$ ordem e Filtro Gaussiano.

$\mathrm{Na}$ figura 4 , podem ser vistas as saídas do estimador de frequência $(\bar{\omega})$ baseadas num filtro Butterworth de $2^{\mathrm{a}}$ ordem e num filtro Gaussiano.
Uma vez obtida a estimativa da frequência de entrada $\bar{\omega}$, deve-se definir um critério para adaptar o ganho do filtro digital para se obter um melhor desempenho. Na seção seguinte, propõe-se um critério de ganho para se obter convergência rápida.

\section{CONVERGÊNCIA}

Se a função definida na equação 16 representa um mapa contrativo $\phi \mapsto g(\phi), \phi \in \mathbb{R}$, então, existe uma constante $L: 0 \leq L<1$, tal que (Blum, 1972):

$$
\left\|g(\phi)-g\left(\phi^{*}\right)\right\| \leq L\left\|\phi-\phi^{*}\right\|,
$$

sendo que $\phi^{*}$ é um ponto fixo.

Portanto o erro, em cada interação é de primeira ordem, ou seja, depende linearmente do erro na interação anterior. No entanto, é possivel se obter uma convergência que dependa do quadrado do erro na interação anterior, mas para isso é preciso enunciar o seguinte teorema, cuja prova pode-se encontrar em (Ortega, 1990):

Teorema 1 Seja $G: \mathbb{R}^{n} \rightarrow \mathbb{R}^{n}$ e suponha que:

\section{G é contínuo e diferenciável no conjunto convexo $D \subset$} $\mathbb{R}^{n}$.

2.

$$
\left\|G^{\prime}(u)-G^{\prime}(v)\right\|<\gamma\|u-v\| \quad \forall u, v \in D .
$$

Então, para todo $x, y \in D$,

$$
\left\|G(y)-G(x)-G^{\prime}(x)(y-x)\right\|<\frac{1}{2} \gamma\|x-y\|^{2} .
$$

Supondo que $\lim _{m \rightarrow \infty} G\left(x_{m}\right)=x^{*} e G^{\prime}\left(x^{*}\right)=0$;

$$
\left\|G\left(x_{m}\right)-G\left(x^{*}\right)\right\|<\frac{1}{2} \gamma\left\|x_{m}-x^{*}\right\|^{2} .
$$

Ou, para interações sucessivas:

$$
\left\|x_{m+1}-x^{*}\right\|<\frac{1}{2} \gamma\left\|x_{m}-x^{*}\right\|^{2} .
$$

Com $\gamma$ escolhido de forma a satisfazer a equação 36.

A partir da equação 38 , pode ser visto que a taxa de convergência diminui segundo o quadrado do erro no passo anterior (perto do ponto fixo o erro é inferior a um).

Então, para a equação 16 define-se o critério de ganho para convergeência rápida, como sendo aquele que estima o valor de $K_{1}^{\prime}$ satisfazendo a condição:

$$
g^{\prime}\left(\phi_{s s}\right)=0 .
$$




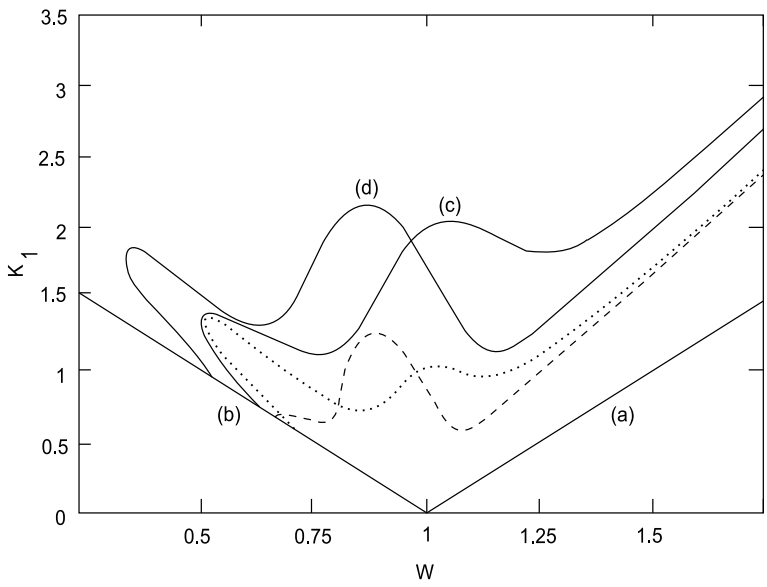

Figura 5: Faixas de captura e curvas de convergência para o TDTL de primeira ordem. A região delimitada pelas curvas (a), (b) e (c) corresponde a $\psi_{0}=\pi / 2$, a região delimitada pelas curvas (a), (b) e (d) corresponde a $\psi_{0}=\pi / 3$. A curva puntilhada corresponde ao ganho para convergência rápida, para $\psi_{0}=\pi / 2$, a curva tracejada corresponde a $\psi_{0}=\pi / 3$.
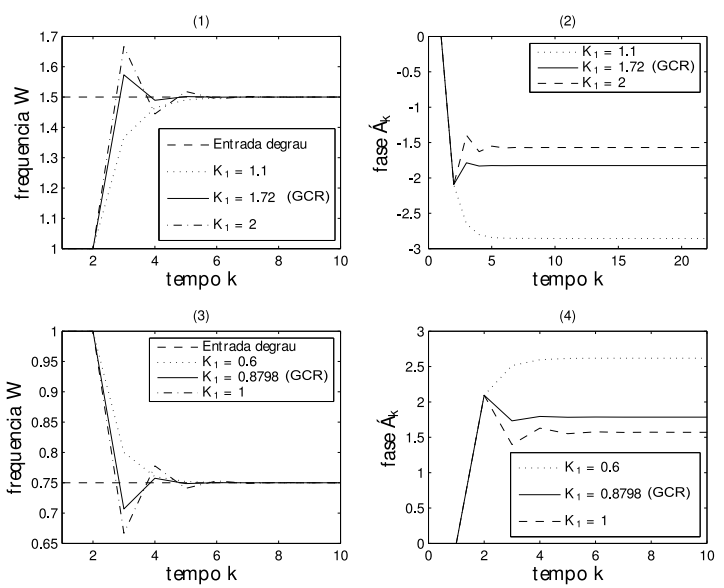

Figura 6: Resposta do TDTL de $1^{\text {a }}$ ordem para entrada degrau com diferentes ganhos. (1) e (2) para $W=1,5$; (3) e (4) para $W=0,75$. GCR: Ganho para convergência rápida.

A condição 40 garante convergência ao ponto fixo.

Faixas de captura e curvas de convergência rápida para o TDTL de $1^{\mathrm{a}}$ ordem, são mostradas na figura 5. Como exemplo, na figura 6 pode-se ver a resposta de um TDTL de $1^{\text {a }}$ ordem com $\psi=\pi / 2$ em dois casos: no primeiro, figuras 6-(1) e 6-(2), com uma entrada tipo degrau degrau de frequência em $W=1,5$ (frequência normalizada); no segundo, figuras 6-(3) e 6-(4), com $W$ igual a 0,75 . Em cada caso, três ganhos foram escolhidos, um deles calculado segundo a condição 40.

\section{ESTIMATIVA DE GANHO}

Como se observou na seção 4, é possível obter uma aproximação $\bar{\omega}$ da frequência de entrada $\omega$, para valores de $\psi_{0}$ no intervalo $(0, \pi / 2]$. Na seção 5 , estabeleceu-se o critério de ganho para se obter convergência rápida.

A estimativa de ganho proposta para o EES-ATDTL é, portanto, um mapa $\bar{\omega} \rightarrow G_{1}$, que associa a cada frequência estimada $\bar{\omega}$, um ganho $G_{1}$, tal que a convergência ao ponto fixo seja proporcional ao quadrado do erro na interação anterior, o que é possível devido a que o ganho $K_{1}=\omega_{0} G_{1}$ é escolhido para satisfazer a condição 40 .

Portanto, a convergência, assim obtida, é mais rápida em relação ao tempo de aquisição.

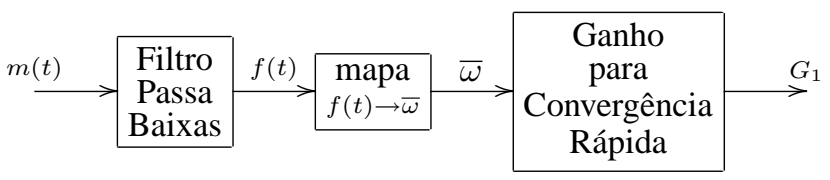

Figura 7: Diagrama em blocos da estimativa de ganho.

Na figura 7, é apresentada a estimativa de ganho proposta. O mapa $f(t) \rightarrow \bar{\omega}$ é definido segundo a equação 34 e o ganho é calculado segundo a condição 40 , sendo $G_{1}$ o ganho do filtro digital. Sempre que ocorrer uma mudança na frequência do sinal de entrada, a estimativa de ganho proposta mudará o ganho do filtro digital, no sentido de obter uma convergência rápida.

\section{RESULTADO DAS SIMULAÇÕES}

Para testar o desempenho do EES-ATDTL com estimação de frequência e convergência rápida, usa-se um sinal FSK (Frequency-shift keying) tal que as frequências de entrada normalizadas sejam $W=\{0,76 ; 1 ; 1,25 ; 1,52\}$, com frequência angular central de livre curso $\omega_{0}$ igual a 1 (rad/seg) e $\psi_{0}$ igual a $\pi / 2 \mathrm{rad}$.

Na figura 8 observa-se que, para $K_{1}=0,5$ os pontos $a$ e $b$ estão dentro da faixa de captura do TDTL, enquanto os pontos $c$ e $d$ estão fora. Os pontos $a 1, b 1, c 1, d 1$ correspondem ao ganho estimado para obter convergência rápida para essas frequências.

Sob tais condições, obtém-se a resposta de um TDTL de $1^{\text {a }}$ ordem com ganho $K_{1}$ fixo e igual a 0,5 . Esse ganho é escolhido para se obter uma boa relação sinal-ruído (Hussain e Boashash, 2001). Obtém-se, também, a resposta do EESTDTL com a estimação de frequência e a convergência rápida propostas. Os resultados obtidos, apresentados na fi- 


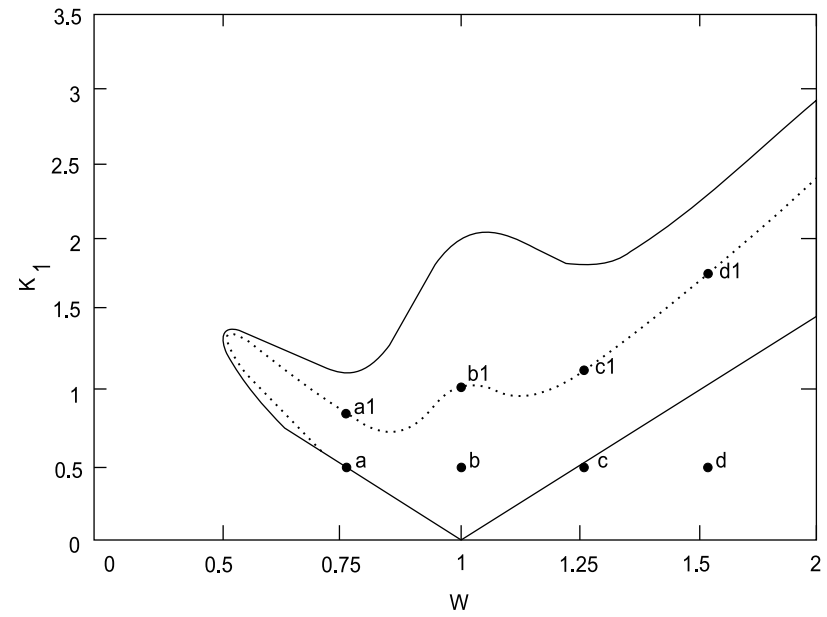

Figura 8: Faixa de captura y curva de convergência rápida para o TDTL com $\psi_{0}=\pi / 2$.

gura 9, mostram que o EES-ATDTL tem uma resposta mais rápida em relação ao TDTL convencional.

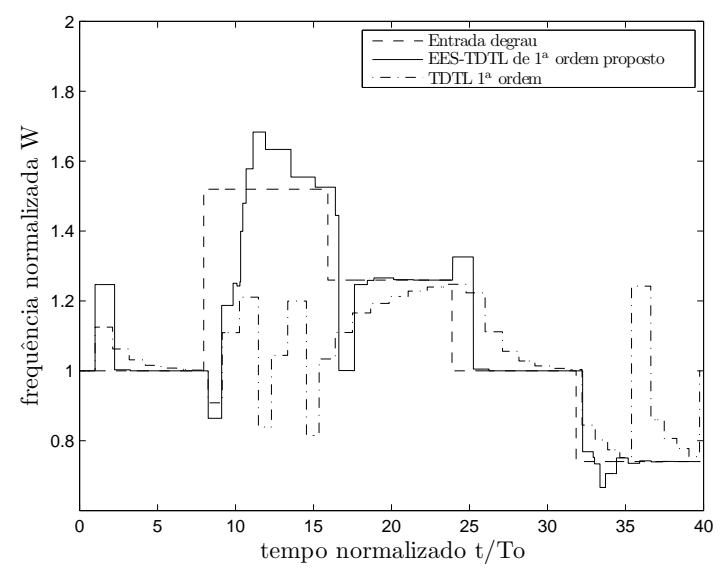

Figura 9: Resposta à entrada degrau para um TDTL de $1^{\text {a }}$ ordem simples e um EES-ATDTL de 1ª ordem com estimação de frequência e convergência rápida.

\section{CONCLUSÕES}

É possivel obter uma estimativa da frequência de entrada a partir dos sinais $x(t)$ e $y(t)$, sempre que $0<\psi_{0} \leq \pi / 2$. Também, é possível, fazendo uso dos teoremas de ponto fixo, calcular um ganho do filtro digital para cada frequência estimada, tal que a convergência ao ponto fixo seja mais rápida.

As simulações mostram que o EES-ATDTL proposto, com estimação de frequência e convergência rápida, oferece uma melhoria significativa no tempo de aquisição, quando comparado com o TDTL simples.

Com a arquitetura proposta, também é possível o aumento da robustez do TDTL, no sentido de tolerar degraus de frequência de maior amplitude, dado que a malha é capaz de adaptar o seu ganho à frequência de entrada.

Com as mesmas considerações apresentadas pode-se, também, ajustar o retardo $\tau$ para obter uma maior faixa de captura, por exemplo, para frequências $W<0,6$ (ver Fig. 2). Nesse caso, além da adaptação do ganho do filtro, pode-se chavear o atraso $\tau$, para mudar o valor de $\psi_{0}$ entre duas desfasagens fixas, por exemplo $\pi / 2$ e $\pi / 3$.

Com os TDTL de $1^{\mathrm{a}}$ ordem é possível obter sincronismo em frequência com erro de fase constante, não nulo. Entretanto, uma análise similar e com as mesmas considerações pode ser feita caso seja necessário sincronismo de fase e frequência, usando um TDTL de $2^{\mathrm{a}}$ ordem, cuja característica principal é ter erro de fase nulo no estado estacionário.

\section{AGRADECIMENTOS}

Este trabalho foi desenvolvido no Laboratorio de Automação e Controle da Escola Politécnica da Universidade de São Paulo com o financiamento da CAPES, do CNPq e da FAPESP.

\section{REFERÊNCIAS}

Akre and, J., Juillard, J., Galayko, D. e Colinet, E. (2010). Synchronized state in networks of digital phase-locked loops, NEWCAS Conference (NEWCAS), 2010 8th IEEE International, pp. 89 -92.

Al-Araji, S., Al-Qutayri, M. e Al-Zaabi, A. (2006). Adaptive TDTL with enhanced performance using sample sensing technique, Circuits and Systems, 2006. ISCAS 2006. Proceedings. 2006 IEEE International Symposium on, p. 4 pp.

Al-Zaabi, A., Al-Qutayri, M. e Al-Araji, S. (2005). Nonuniform sampling digital PLL with fast error correction technique, Electronics, Circuits and Systems, 2005. ICECS 2005. 12th IEEE International Conference on, pp. $1-4$.

Best, R. E. (2003). Phase-Locked Loops, Design, Simulations, and Applications, McGraw-Hill Book Company.

Blum, E. K. (1972). Numerical Analysis and Computation Theory and Practice, Reading, Mass., Addison-Wesley Pub. Co. 
Bueno, A., Ferreira, A. A. e Piqueira, J. R. C. (2009). Fully connected PLL networks: How filter determines the number of nodes, Mathematical Problems in Engineering 2009: 13.

Bueno, A., Ferreira, A. A. e Piqueira, J. R. C. (2010). Modeling and filtering double-frequency jitter in one-way master-slave chain networks, Circuits and Systems I: Regular Papers, IEEE Transactions on PP(99): 1 -8.

Bueno, A., Rigon, A. e Ferreira, A. (2010). Design constraints for third-order PLL nodes in master-slave clock distribution networks, Communications in Nonlinear Science and Numerical Simulation 15(9): 2565 - 2574.

Carareto, R., Orsatti, F. M. e Piqueira, J. R. (2009). Reachability of the synchronous state in a mutually connected PLL network, AEU - International Journal of Electronics and Communications 63(11): 986 - 991.

Hussain, Z. e Boashash, B. (2001). Statistical analysis of the time-delay digital tanlock loop in the presence of gaussian noise, Circuits and Systems, 2001. ISCAS 2001. The 2001 IEEE International Symposium on, Vol. 4, pp. $21-24$ vol. 4.

Hussain, Z., Boashash, B., Hassan-Ali, M. e Al-Araji, S. (2001). A time-delay digital tanlock loop, Signal Processing, IEEE Transactions on 49(8): 1808-1815.

Lee, J. e Un, C. (1982). Performance analysis of digital tanlock loop, Communications, IEEE Transactions on 30(10): 2398-2411.

Lindsey, W. e Chie, C. M. (1981). A survey of digital phaselocked loops, Proceedings of the IEEE 69(4): 410-431.

Monteiro, L., Dos Santos, V. e Piqueira, J. (2003). Estimating the critical number of slave nodes in a single-chain PLL network, Communications Letters, IEEE 7(9): 449 -450 .

Monteiro, L., Garcia, P. e Piqueira, J. (2003). Sincronismo em redes mestre-escravo com atraso, SBA: Controle \& Automação - Sociedade Brasileira de Automatica 14: $121-126$.

Orsatti, F., Carareto, R. e Piqueira, J. (2008). Mutually connected phase-locked loop networks: dynamical models and design parameters, IET Circuits, Devices \& Systems 2(6): 495-508.

Ortega, J. M. (1990). Numerical Analysis: A Second Course, Philadelphia : Society for Industrial and Applied Mathematics.

Osborne, H. (1980). Stability analysis of an nth power digital phase-locked loop-part i: First-order DPLL, Communications, IEEE Transactions on 28(8): 1343-1354.
Piqueira, J. R. C. (1997). Uma Contribução ao estudo das redes com malhas de sincronismo de fase, Universidade de São Paulo, Escola Politécnica. Tese Livre-Docência.

Popken, L., Kriedte, W., Bender, O. e Luise, M. (1993). Synchronization failure rates in master-slave synchronized phase-locked loops, Aerospace and Electronic Systems, IEEE Transactions on 29(2): 328 -335.

Saleh R. Al-Araji, Z. M. H. e Al-Qutayri, M. A. (2006). Digital phase lock loops : architectures and applications, Springer, Dordrecht.

Vamvakos, S. D., Staszewski, R. B., Sheba, M. e Waheed, K. (2006). Noise analysis of time-to-digital converter in all-digital PLLs, Design, Applications, Integration and Software, 2006 IEEE Dallas/CAS Workshop on, pp. 87 -90 .

Wiggins, S. (1990). Introduction to Applied Nonlinear Dynamical Systems and Chaos, Springer-Verlag. 